\title{
A LEMMA OF THE THEORY OF LINEAR DIFFERENTIAL SYSTEMS*
}

\author{
BY J. D. TAMARKIN
}

The reading of the preceding note by $W$. M. Whyburn suggests to me a lemma which, although it is simple, deserves to be stated and proved separately. The results of Whyburn $\dagger$ as well as those of Miss Whelan $\ddagger$ may be derived immediately from this lemma.

We shall use the matrix notation and designate by German capitals the square matrices of $n$ rows and $n$ columns, and by small German letters the $n$-dimensional vectors, with the usual agreements as to the products of matrices by matrices or by vectors and so on.

By a solution of a linear differential system of $n$th order with integrable $(L)$ coefficients we shall mean a vector whose components are absolutely continuous and satisfy the system almost everywhere.

LEMmA. Let the coefficients $a_{i j}^{\prime}(x), a_{i j}^{\prime \prime}(x), b_{i}^{\prime}(x), b_{i}^{\prime \prime}(x)$ of the linear differential system

$$
\begin{aligned}
& \frac{d \mathfrak{y}^{\prime}(x)}{d x}=\mathfrak{U}^{\prime}(x) \cdot \mathfrak{y}^{\prime}(x)+\mathfrak{b}^{\prime}(x), \\
& \frac{d \mathfrak{y}^{\prime \prime}(x)}{d x}=\mathfrak{U}^{\prime \prime}(x) \cdot \mathfrak{y}^{\prime \prime}(x)+\mathfrak{b}^{\prime \prime}(x)
\end{aligned}
$$

be integrable on $(0,1)$. Let

$$
\begin{aligned}
& \max _{i}\left\{\sum_{j=1}^{n} \int_{0}^{1}\left|a_{i j}^{\prime}(x)\right| d x\right\}=M_{1}, \\
& \max _{i}\left\{\sum_{j=1}^{n} \int_{0}^{1}\left|a_{i j}^{\prime \prime}(x)\right| d x\right\}=M_{2},
\end{aligned}
$$

* Presented to the Society, October 26, 1929.

$\dagger$ See also $\S 4$ of Whyburn's paper referred to, on p. 94 of this issue.

$\ddagger$ This Bulletin, vol. 35 (1929), pp. 112-119. Our lemma is but a slight modification of and is proved in a much the same way as lemma $v$ (p. 116) of Miss Whelan. 


$$
\begin{aligned}
& \max _{i}\left\{\int_{0}^{1}\left|b_{i}^{\prime}(x)\right| d x\right\}=N_{1}, \\
& \max _{i}\left\{\int_{0}^{1}\left|b_{i}^{\prime \prime}(x)\right| d x\right\}=N_{2}, \\
& \max _{i}\left\{\int_{0}^{1} \sum_{j=1}^{n}\left|a_{i j}^{\prime}(x)-a_{i j}^{\prime \prime}(x)\right| d x\right\}=\epsilon, \\
& \max _{i}\left\{\int_{0}^{1}\left|b_{i}^{\prime}(x)-b_{i}^{\prime \prime}(x)\right| d x\right\}=\eta . \\
& (i=1,2, \cdots, n),
\end{aligned}
$$

Let $\mathfrak{c}$ be a vector with constant components and $\max _{i}\left\{\left|c_{i}\right|\right\}=C$. Then, if $\mathfrak{y}^{\prime}(x), \mathfrak{y}^{\prime \prime}(x)$ are respectively the solutions of (1) and (2) satisfying the initial conditions

$$
\mathfrak{y}^{\prime}(0)=\mathfrak{y}^{\prime \prime}(0)=\mathfrak{c},
$$

we have on $(0,1)$

(4) $\left|y_{i}^{\prime}(x)-y_{i}^{\prime \prime}(x)\right| \leqq\left\{\begin{array}{l}\left(\eta+\epsilon K_{1}\right) e^{M_{2}}, K_{1}=\left(C+N_{1}\right) e^{M_{1}}, \\ \left(\eta+\epsilon K_{2}\right) e^{M_{1}}, K_{2}=\left(C+N_{2}\right) e^{M_{2}}\end{array}\right.$

$$
(i=1,2, \cdots, n) \text {. }
$$

We observe first that the solution of a system

$$
\frac{d \mathfrak{y}(x)}{d x}=\mathfrak{U}(x) \cdot \mathfrak{y}(x)+\mathfrak{b}(x)
$$

with integrable coefficients exists and is uniquely determined by its initial values:

$$
\mathfrak{y}(0)=\mathfrak{c} .
$$

Furthermore, on setting

$$
\max _{i}\left\{\sum_{j=1}^{n} \int_{0}^{1}\left|a_{i j}(x)\right| d x\right\}=M, \max _{i}\left\{\int_{0}^{1}\left|b_{i}(x)\right| d x\right\}=N,
$$

the following inequalities hold:

$$
\left|y_{i}(x)\right| \leqq(C+N) e^{M}, \quad(i=1,2, \cdots, n) .
$$

The proof of these facts is readily obtained by means of the classical method of successive approximations in a slightly 
modified form, due to the non-boundedness of the functions $a_{i j}(x)$ and $b_{i}(x)$. The method of successive approximations must be applied not directly to the system (5) but rather to the equivalent system of integral equations*

$$
\mathfrak{y}(x)=\mathfrak{c}+\int_{0}^{x} \mathfrak{b}(t) d t+\int_{0}^{x} \mathfrak{H}(t) \cdot \mathfrak{y}(t) d t .
$$

Now we can replace systems (1) and (2) with the initial conditions (3) by the equivalent systems of integral equations

$$
\begin{aligned}
& \mathfrak{y}^{\prime}(x)=\mathfrak{c}+\int_{0}^{x} \mathfrak{b}^{\prime}(t) d t+\int_{0}^{x} \mathfrak{Y}^{\prime}(t) \cdot \mathfrak{h}^{\prime}(t) d t, \\
& \mathfrak{y}^{\prime \prime}(x)=\mathfrak{c}+\int_{0}^{x} \mathfrak{b}^{\prime \prime}(t) d t+\int_{0}^{x} \mathfrak{Y}^{\prime \prime}(t) \cdot \mathfrak{h}^{\prime \prime}(t) d t,
\end{aligned}
$$

whence

$$
\mathfrak{h}^{\prime}(x)-\mathfrak{y}^{\prime \prime}(x) \equiv \mathfrak{y}(x)=\int_{0}^{x} \mathfrak{b}(t) d t+\int_{0}^{x} \mathfrak{A}(t) \mathfrak{y}(t) d t
$$

where we have put

and

$$
\mathfrak{b}(x)=\mathfrak{b}^{\prime}(x)-\mathfrak{b}^{\prime \prime}(x)+\left\{\begin{array}{l}
\int_{0}^{x}\left[\mathfrak{H}^{\prime}(t)-\mathfrak{A}^{\prime \prime}(t)\right] \cdot \mathfrak{y}^{\prime}(t) d t \\
\text { or } \\
\int_{0}^{x}\left[\mathfrak{H}^{\prime}(t)-\mathfrak{Y}^{\prime \prime}(t)\right] \cdot \mathfrak{y}^{\prime \prime}(t) d t
\end{array}\right.
$$

$$
\mathfrak{A}(x)=\left\{\begin{array}{l}
\mathfrak{U}^{\prime \prime}(x) \text { or } \\
\mathfrak{H}^{\prime}(x)
\end{array}\right.
$$

Using (7) we get the proof of our lemma.

If inequality (7) is assumed our lemma can be derived from a general existence theorem by Carathéodory. $\dagger$ But this inequality does not follow directly from Carathéodory's discussion. Moreover the method used above presents the advantage of giving an explicit estimate for the difference.

We can leave to the reader the derivation of the results of W. M. Whyburn and of Miss Whelan, as well as the applica-

* Tamarkin, Some general problems of the theory of ordinary linear differential equations, Petrograd, 1917, pp. 29-39 (in Russian).

$\dagger$ Vorlesungen über reelle Funktionen, Teubner, Leipzig, 1927, p. 678. 
tion of this lemma to the case where the coefficients of the system (5) and the initial data (6) depend on a parameter. The interval $(0,1)$ of course can be replaced by an arbitrary interval $(a, b)$, the initial values $\mathfrak{y}^{\prime}(0), \mathfrak{y}^{\prime \prime}(0)$ may be made distinct and the whole theory can be extended to systems of infinitely many equations, under suitable restrictions upon the matrices and vectors involved.

BROWN UNIVERSITY

\title{
ON THE NUMBER OF APPARENT MULTIPLE POINTS OF VARIETIES IN HYPERSPACE
}

\author{
BY B. C. WONG
}

By an apparent point of multiplicity $s$ on a variety in $r$-space we mean a line which passes through a given point in $r$-space and meets the variety in $s$ distinct points. In order that the number of such apparent $s$-fold points on a variety $V_{x}^{m}$ of order $m$ and of $x$ dimensions be finite, we must have $r=s t+1$, $x=(s-1) t$, where $t$ is the number less one of the hypersurfaces intersecting in the variety. In other words, the number of apparent $s$-fold points on a $V_{(s-1) t}^{m}$ which is the intersection of $t+1$ hypersurfaces in $S_{s t+1}$ is finite. It is the purpose of this paper to determine this number and also to determine its upper and lower limits.

We shall use the symbols $H_{s}^{(r)}, \bar{h}_{s}^{(r)}$ to denote respectively the maximum and minimum number of apparent $s$-fold points that a $V_{(s-1) t}^{m}$ of any order $m$ in $S_{r}[r=s t+1]$ can have, and the symbol $h_{s}^{(r)}$ to denote the number of those that a $V_{(s-1) t}^{m}$ of order $m=n_{1} n_{2} \cdots n_{t+1}$, which is the complete intersection of $t+1$ hypersurfaces of orders $n_{1}, n_{2}, \cdots, n_{t+1}$ respectively, ordinarily has. Thus if $s=2, t=1$ and therefore $r=3$, a curve $C^{m}$ in $S_{3}$ can have at most

$$
H_{2}{ }^{(3)}=(m-1)(m-2) / 2,
$$

and at least

$$
\bar{h}_{2}^{\left({ }^{(3)}\right.}=m(m-2) / 4, \quad \text { for } m \text { even, }
$$

and

$$
\bar{h}_{2}^{(3)}=(m-1)^{2} / 4, \quad \text { for } m \text { odd },
$$

